\title{
Intensity-modulated particle beam radiation therapy in the management of olfactory neuroblastoma
}

\author{
Weixu Hu ${ }^{1,2}$, Jiyi Hu ${ }^{1,2}$, Jing Gao ${ }^{1,2}$, Jing Yang ${ }^{1,2}$, Xianxin Qiu ${ }^{1,2}$, Lin Kong ${ }^{2,3}$, Jiade J. Lu ${ }^{1,2}$ \\ ${ }^{1}$ Department of Radiation Oncology, Shanghai Proton and Heavy Ion Center, Shanghai, China; ${ }^{2}$ Shanghai Engineering Research Center of Proton \\ and Heavy Ion Radiation Therapy, Shanghai, China; ${ }^{3}$ Department of Radiation Oncology, Shanghai Proton and Heavy Ion Center, Fudan University \\ Cancer Hospital, Shanghai, China \\ Contributions: (I) Conception and design: W Hu, L Kong, JJ Lu; (II) Administrative support: L Kong, JJ Lu; (III) Provision of study materials or \\ patients: W Hu, J Hu, J Gao, L Kong, JJ Lu; (IV) Collection and assembly of data: W Hu, J Hu, J Yang, X Qiu, L Kong, JJ Lu; (V) Data analysis and \\ interpretation: W Hu, J Hu, L Kong, JJ Lu; (VI) Manuscript writing: All authors; (VII) Final approval of manuscript: All authors. \\ Correspondence to: Lin Kong, MD; Jiade J. Lu, MD, MBA. Shanghai Proton and Heavy Ion Center, 4365 Kangxin Road, Pudong, Shanghai 201321, \\ China. Email: lin.kong@sphic.org.cn; jiade.lu@sphic.org.cn.
}

\begin{abstract}
Background: To report the clinical experience and short-term efficacy in the management of olfactory neuroblastoma (ONB).

Methods: We performed a retrospective analysis of $12 \mathrm{ONB}$ patients treated with particle beam radiation therapy (PBRT) between 12/2015 and 5/2019 at the Shanghai Proton and Heavy Ion Center. Four (33.3\%) patients presented with Kadish B ONB, and 8 (66.7\%) presented with Kadish C or D disease. Eleven patients received proton radiotherapy (PRT) followed by a carbon ion radiotherapy (CIRT) boost, one patient received CIRT only. The 2-year survival rates were calculated using the Kaplan-Meier method. Acute and late adverse events were summarized and scored according to the CTCAE (version 4.03).

Results: With a median follow-up of 17.5 (range, 2.53-49.9) months, all patients but 1 were alive. Eight patients were alive without evidence of disease, and 2 additional patients achieved partial response and remained alive with residual disease. One patient died of toxicity associated with salvage chemotherapy for distant metastasis and local failure. Another patient developed distant metastasis only and was alive at the time of the last follow-up. The 2-year OS, PFS, LRPFS, and DMFS rates were $83.3 \%, 75.8 \%, 87.5 \%$, and $79.5 \%$, respectively. No acute or late toxicities of $\geq$ grade 3 was observed.

Conclusions: Intensity modulated PBRT of ONB is well tolerated. While longer follow-up is needed, early outcomes suggested that PBRT is safe and effective for the treatment of ONB with minimal adverse events.
\end{abstract}

Keywords: Esthesioneuroblastoma; olfactory neuroblastoma (ONB); radiotherapy; carbon ion radiation; proton beam radiation

Submitted Dec 31, 2019. Accepted for publication Jul 09, 2020.

doi: 10.21037/atm-19-4790

View this article at: http://dx.doi.org/10.21037/atm-19-4790

\section{Introduction}

Olfactory neuroblastoma (ONB), also known as esthesioneuroblastoma, is an uncommon malignancy arises from the olfactory neuroepithelium. ONB accounts for merely $3-6 \%$ of all sinonasal malignancies (1-4), although the incidence increased sharply over the past decades. The optimal strategy for the management of ONB is yet to be determined as the rarity of the disease precluded prospective randomized clinical trials and large-scale retrospective investigations. ONB is typically anatomically complex because of the critical organs at risk (OARs) nearby, and complete surgical resection with sufficient margins is usually 
difficult to achieve. Results from a few retrospective studies suggested that surgery with postoperative radiotherapy significantly improved local control and overall survival as compared with monotherapy using surgery or radiotherapy alone $(2,4-9)$. As such, multidisciplinary approach including surgery and radiotherapy (with or without induction and/ or concurrent chemotherapy) has been advocated $(8,10)$. For patients with unresectable, inoperable, or incompletely resected $\mathrm{ONB}$, high-dose radiation therapy may provide an opportunity of cure or long-term disease control (8). Nevertheless, the dose constrains of the OARs adjacent to the gross tumor may limit the radiation dose delivered to the target volume(s).

Accelerated beams of charged particles (e.g., proton and carbon ion) are featured with a finite range and a distant Bragg peak. Dosimetry studies have demonstrated that particle beam radiation therapy (PBRT) enables the delivery of high-dose radiation to the target volume(s) while sparing OARs thereby enhancing the therapeutic ratio over photon-based intensity-modulated radiotherapy (IMRT) in patients with tumors of the base of skull (11-13). The use of intensity modulated PBRT further provides more precise and conformal dose distribution $(14,15)$. In addition to the more advanced dosimetric characteristic of PBRT, as a high linear energy transfer (LET) beam, carbon-ion beam possesses higher relative biological effectiveness (RBE) as compared to proton or photon (16-19). As such, carbon-ion radiotherapy (CIRT) may induce more effective cell killing in theory.

Despite of the theoretical advantages of PBRT in the management of skull base tumors, clinical evidence on the utilization of PBRT for ONB is scarce and is usually limited to small case series. The purpose of this study is to bolster the existing literature by document an additional group of ONB patients definitively treated with PBRT at the Shanghai Proton and Heavy Ion Center (SPHIC).

We present the following article in accordance with the STROBE reporting checklist (available at http://dx.doi. org/10.21037/atm-19-4790).

\section{Methods}

\section{Patients characteristics and pretreatment evaluation}

Between 12/2015 and 5/2019, 13 consecutive patients with histologically confirmed ONB were treated at SPHIC. The study was conducted in accordance with the Declaration of Helsinki (as revised in 2013). This retrospective study was approved by the institutional review board (IRB) of the SPHIC (IRB No. 191205EXP-01). All patients obtained written informed consent before enrolling in this study.

One patient received palliative PBRT for laryngeal and lymph node metastasis in the lower neck was excluded from this retrospective analysis. Among the remaining patients, 10 were males and 2 were females. The median age was 40 (range, 14-77) years. Pretreatment baseline evaluations included a complete history and physical examination, complete blood count, serum electrolytes, hepatic and renal function tests, and EKG. MRI or CT (if MRI was contraindicated) of the head and neck region is mandatory for all patients. Whole body FDG-PET/CT was preferred to rule out distant metastasis; however, CT of the thorax, ultrasound or CT of the abdomen, and bone scan were used if PET/CT is contraindicated or declined. Both Kadish and AJCC staging system $\left(7^{\text {th }}\right.$ or $8^{\text {th }}$ edition depend on the date of pathological diagnosis) were used. Eight (66.7\%) patients presented with Kadish C or D disease, and 4 (33.3\%) patients had Kadish B disease. Direct invasion to the brain or dura mata presented in 7 (58.3\%) patients at diagnosis. One patient presented with $\mathrm{N} 1$ and another had N3 disease at diagnosis.

The characteristics of all 12 patients and their diseases were detailed in Table 1. All cases were discussed in the multidisciplinary tumor clinic of SPHIC to confirm the indication of intensity modulated PBRT before their inclusion into our institutional cancer registry and treatment planning.

\section{Surgery and chemotherapy}

Nine patients underwent surgery, including 8 had R2 resection with gross residual tumor and 1 achieved R 1 resection. Three patients underwent biopsy only. Induction chemotherapy was used under instruction of the medical oncologists. Among the 7 patients received induction chemotherapy, 2 achieved partial response (PR), and 5 had stable disease (SD). As such, 11 patients $(91.7 \%)$ had gross disease prior to PBRT with a median volume of $44.7 \mathrm{~mL}$ (range, 20.29-234.19). Concurrent chemotherapy with platinum-based regimens was used in 6 patients at the discretion of the medical oncologists. No patient received adjuvant chemotherapy after PBRT.

\section{PBRT: Immobilization and definition of target volumes}

All patients were immobilized in supine position with 
Table 1 Characteristics of the 12 patients with non-metastatic ONB

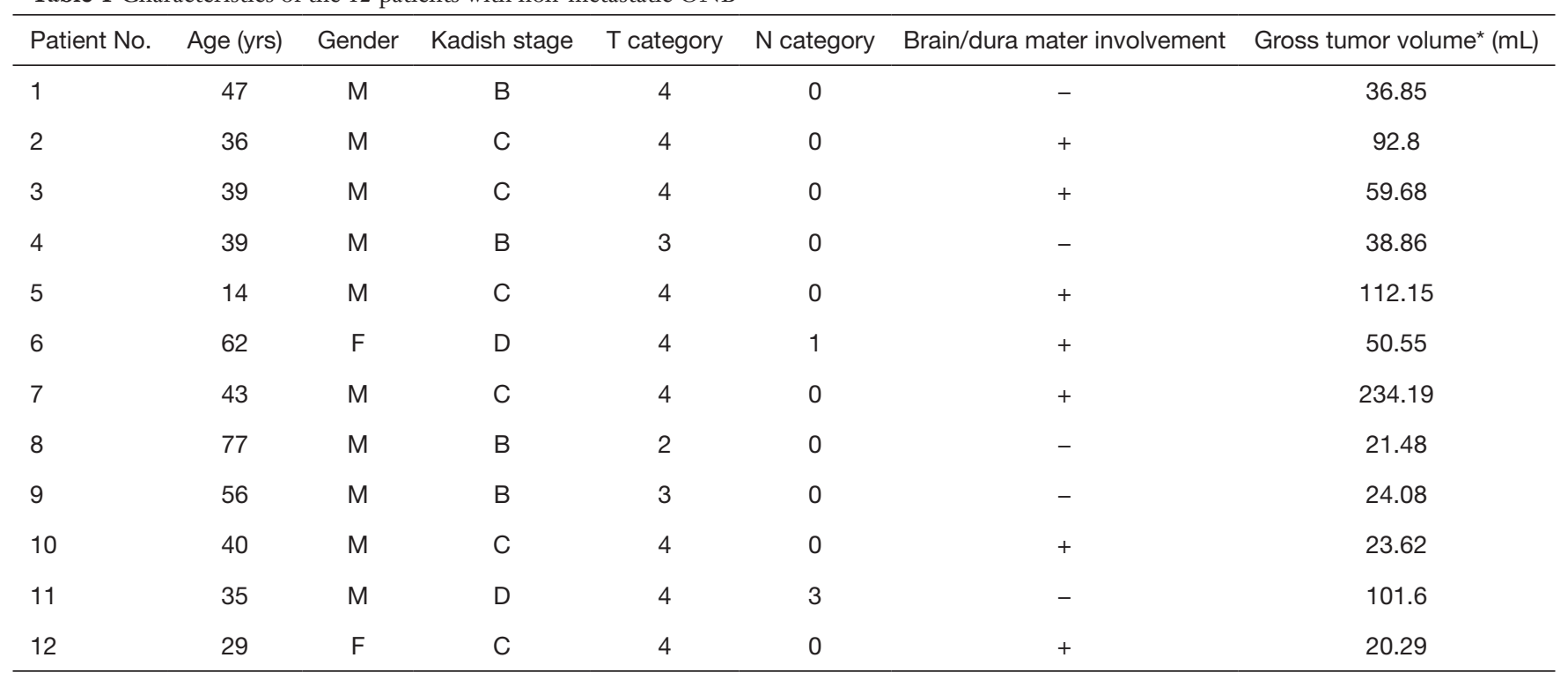

${ }^{*}$, gross tumor volume represents the tumor volume before particle beam radiotherapy. M, male; F, female.

AlphaCradle ${ }^{\circledR}$ and thermoplastic masks. CT scans without intravenous contrast from the vertex to the inferior margin of clavicular heads at $1.5-\mathrm{mm}$ slice thickness were performed for simulation. MRI-CT fusion was performed for all patients for target delineation. The gross tumor volume (GTV) including all disease observed on clinical examination or imaging studies for patients with incomplete surgical resection or biopsy at the primary site (GTVp) or positive lymph nodes (GTVn). We defined a clinical target volume of GTVp (CTV-G) or GTVn (CTV-N) as GTVp or GTVn with a $3 \mathrm{~mm}$ margin (limited to $1 \mathrm{~mm}$ near OARs). The CTVs encompasses both pretreatment tumor bed plus high-risk areas for tumor extension for patients with $\mathrm{R} 1$ resection or achieved complete response (CR) after chemotherapy. Elective nodal irradiation (ENI) was provided to 8 patients. The planning target volume (PTV) was CTVs with a 3-6 mm margin for uncertainty with regard to dose distribution and potential setup errors.

\section{PBRT: Treatment planning and dose prescription}

The Syngo ${ }^{\circledR}$ treatment planning system (Siemens, Erlangen, Germany) was used for planning of PRT and CIRT. Two or 3 beams were typically used for treatment plans. Doses of PBRT were prescribed in Gy (RBE). Dose constraints of critical OARs were based on TD5/5 described by Emami et al. (20). Experience from the National Institute of Quantum and Radiation Science, Japan (NIQRS) was used for the CIRT dose constraints of optic nerves (D20<30 GyE) and temporal lobes (V40<7.66 cc; V50<4.66 cc) (21). All patients received intensity modulated PBRT with pencil beam scanning (PBS) technology. Weekly CT were performed in all patients to ensure dose distribution.

Ten patients received PRT to 54-56 Gy (RBE), in 2830 daily fractions, followed by a CIRT boost to $15-17.5$ Gy (RBE) in 5 daily fractions. One patient received CIRT only to $66 \mathrm{GyE}$ in 22 daily fractions. The only patient who achieved R1 resection without gross disease received PRT to 56 Gy (RBE) in 28 fractions followed by a CIRT boost of 10.5 Gy (RBE) in 3 fractions. One adolescent patient (14 years old) received $54 \mathrm{~Gy}$ (RBE) in 30 fractions of PRT followed by CIRT boost to 15 Gy (RBE) in 5 fractions due to constrains to the OARs. Two patients with neck adenopathy also received intensity modulated PBRT to the neck. Six additional patients deemed to have high risk for neck metastasis received elective cervical irradiation. All patients completed PBRT without unplanned break.

The details of treatment received including the use of surgery and chemotherapy as well as the dose and fractionation of PBRT were detailed in Table 2 for each 
Table 2 Treatment received and outcomes of the 12 patients with non-metastatic ONB

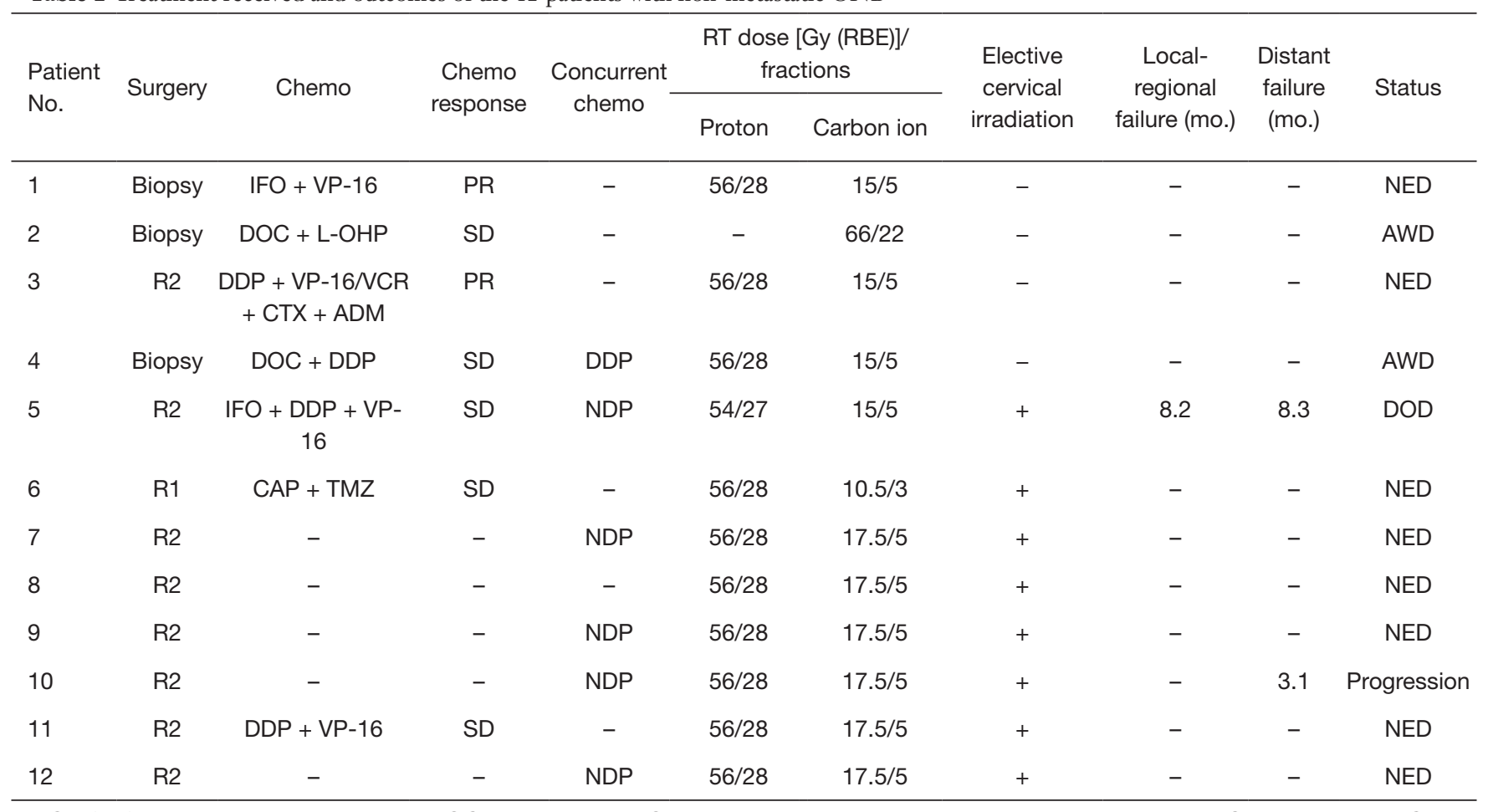

IFO, ifosfamide; VP-16, etoposide; DOC, docetaxel; L-OHP, oxaliplatin; DDP, cisplatin; NDP, nedaplatin; VCR, vincristine; CTX, cyclophosphamide; ADM, adriamycin; CAP, capecitabine; TMZ, temozolomide; PR, partial response; SD, stable disease; RT, radiotherapy; GyE, Gy-equivalents; NED, no evidence of disease; DOD, died of disease; AWD, alive with disease.

individual patient.

\section{Follow-up}

All patients were required to be followed-up according to our institutional follow-up protocol. The first followup was scheduled at 4-6 weeks after the completion of PBRT. Patients were then followed-up every 3-4 months within first 2 years, every 6 months in the following 3 years, and annually thereafter. A complete history and physical examination with a focus to the head and neck region, blood tests (complete blood count (CBC), electrolytes, liver function test (LFT), and renal function test (RFT)), MRI or CT scans of the head and neck were required at each follow-up. Other studies such as CT of the thorax, CT or ultrasound of the abdomen, whole body FDG-PET/CT, are ordered if clinically indicated.

\section{Data analysis}

The duration of survival was calculated from the diagnosis of the disease until death or the date of last follow-up. The time to local, regional, and/or distant failure was measured from the initiation of any treatment until recurrence or disease progression. Rates of overall survival (OS), progression free survival (PFS), locoregional failure free survival (LRPFS), distant metastasis free survival (DMFS) were calculated using the Kaplan-Meier method. All analyses were performed using the SPSS statistics package (Version 25.0).

Adverse events were defined and scored according to the CTCAE (version 4.03). Acute toxicities included the those occurred during or within 3 months after the initiation of PBRT. Late toxicity was defined as those occurred after 3 months from or persisted for $>3$ months after the completion of PBRT.

\section{Results}

\section{Disease control and survival}

Between 12/2015 and 5/2019, 13 consecutive patients with histologically confirmed ONB were treated at SPHIC, one 
A

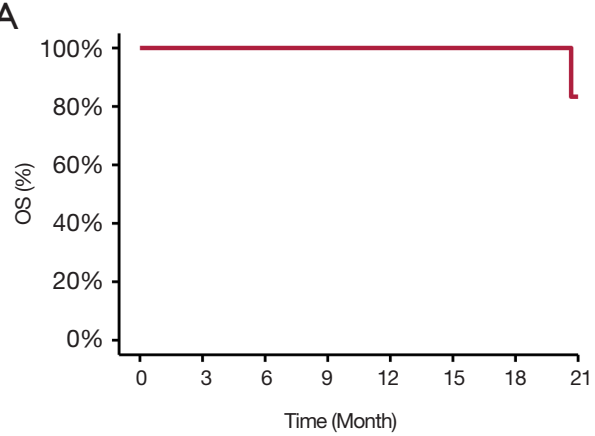

B

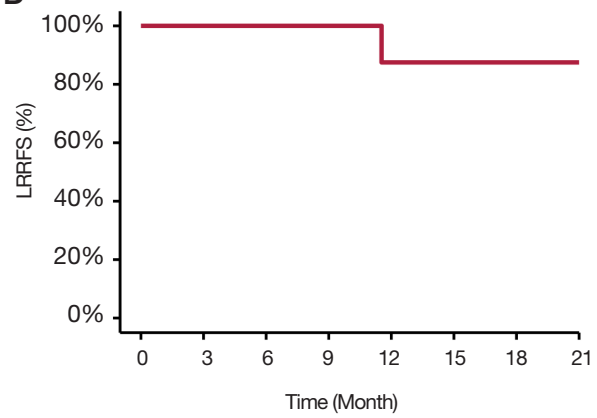

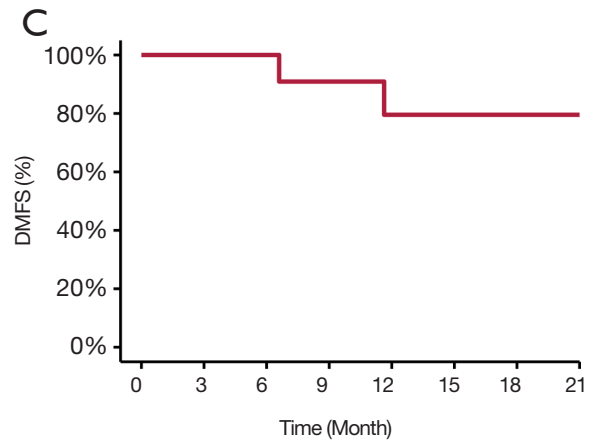

$\mathrm{D}$

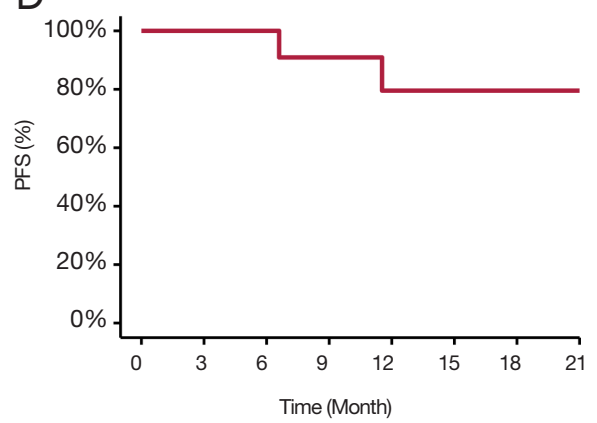

Figure 1 Overall survival (OS) (A), local-regional recurrence-free survival (B), distant metastasis free-survival (DMFS) (C), and progressionfree survival (PFS) (D) rate curves of the entire cohort.

patient received palliative PBRT for laryngeal and lymph node metastasis in the lower neck was excluded from this retrospective analysis. With a median follow-up of 17.5 (range, 2.53-49.9) months, all patients but 1 were alive, and 8 patients without evidence of disease. Two patients who achieved partial response remain alive with disease. One patient with Kadish C disease developed distant recurrence at 8.2 months after the completion of PBRT, then local recurrence 3 months later. The patient received salvage chemotherapy and immunotherapy but succumbed from toxicities of chemotherapy 17 months after the completion of PBRT. Another patient developed liver metastasis at 3.1 months after PBRT without local or regional recurrence. The patient received salvage chemotherapy and was alive at the time of this analysis. The 2-year OS, PFS, LRPFS, and DMFS rates were $83.3 \%, 75.8 \%, 87.5 \%$, and $79.5 \%$, respectively (Figure 1). Treatment characteristics and outcomes were detailed in Table 2.

\section{Radiation-induced toxicities}

Characteristics of acute and late toxicities are summarized in Table 3. No acute toxicities of grade 3 or above was observed in any of the 12 patients. Mild skin erythema was the most commonly observed toxicity during PBRT and was developed in $11(91.7 \%)$ patients. Grade 1 or 2 mucositis and xerostomia were also observed in $7(58.3 \%)$ and 4 (33.3\%) of patient, respectively. Two patients remained to experience mild xerostomia after the completion of PBRT. Xerostomia of these 2 patients persisted for more than 3 months after the completion of PBRT, 1 with grade 1 and another with grade 2 . No other late adverse effect was observed.

\section{Discussion}

The results of this retrospective analysis of 12 patients with ONB revealed that intensity modulated PBRT using pencil beam scanning (PBS) technology produced favorable 2-year outcomes in terms of OS, disease control, and toxicity profile. Only one patient developed distant metastasis followed by locoregional recurrence after PBRT, and another developed distant metastasis alone. With a median follow-up time of 17.5 months, the 2-year OS, PFS, LRPFS, and DMFS rates were $83.3 \%, 75.8 \%, 87.5 \%$ and $79.5 \%$, respectively. Distant metastasis was the main 
Table 3 Characteristics of acute and late toxicities

\begin{tabular}{|c|c|c|c|c|c|c|c|c|}
\hline $\begin{array}{l}\text { Type of adverse } \\
\text { reaction }\end{array}$ & \multicolumn{4}{|c|}{ Acute toxicities } & \multicolumn{4}{|c|}{ Late toxicities } \\
\hline Skin erythema & $9(75.0)$ & $2(16.7)$ & 0 & $11(91.7)$ & 0 & 0 & 0 & 0 \\
\hline Mucositis & $6(50.0)$ & $1(8.3)$ & 0 & 7 (58.3) & 0 & 0 & 0 & 0 \\
\hline
\end{tabular}

mode of treatment failure particularly in patients with locoregionally advanced disease. In addition, despite of the definitive PBRT dose delivered, no patient experienced radiation-induced severe (i.e., $\geq$ grade 3 ) acute or late toxicities.

Kadish staging system was one of the most widely utilized clinical staging system for ONB, which was first proposed by Kadish et al. (22), and later modified by Morita et al. (23). Kadish staging has been identified as significant prognosis factor for survival. The 5-year OS rates of patients with Kadish $\mathrm{A} / \mathrm{B}$ ONB is close to $90 \%$, but between $50-70 \%$ in Kadish C/D disease (24-29). In addition to Kadish stage, an analysis of National Cancer Database (NCDB), patients with large gross tumor of $>4 \mathrm{~cm}$ in diameter had increased risk of death after definitive dose of radiation therapy (27). Most of the patients in the current study had advanced disease at presentation including $8(66.7 \%)$ had stage C/D and $4(33.3 \%)$ with stage $\mathrm{B}$ disease. In addition, all but one patient had R2 resection or biopsy, and only one patient achieved R1 resection. The median volume of GTV in our patients was $44.7 \mathrm{~mL}$ (range, 20.29-234.19 mL). After PRT followed by CIRT boost or CIRT alone, only 1 patient with stage $\mathrm{C}$ who experienced $\mathrm{R} 2$ resection with a residual tumor of $112.2 \mathrm{~mL}$ developed locoregional progression following distant metastasis. Another patient with stage C disease who had debulking achieved PR after completion of PBRT, experienced distant metastasis at 3.1 months.

Although no standard single or multi-modality treatment has been confirmed for ONB, surgery remains the mainstay treatment. However, due to the complex anatomic location of the disease, complete surgical resection is usually not feasible in patients with locally advanced disease. Surgery alone, with a 5-year overall survival of 38-68\%, is considered insufficient for effective disease control especially for patients with Kadish stage B-D disease $(2,9,30,31)$. The risk of local failure is close to $50 \%(6,29)$, and the 5 -year disease free survival (DFS) rate is merely $\sim 40 \%$ in patients with positive surgical margin or gross residual tumor $(6,29,32)$. Therefore, a multidisciplinary approach including surgery and radiotherapy has been advocated $(8,10,29,33,34)$. And various researches showed that surgery in combination with radiotherapy could improve the OS rates to $70-90 \%(6-9,28,35)$ and increased LPFS rates to $50-90 \%(6-8,35)$. In a paper from our research group that has yet to be published reported that the 3-year local and regional recurrence-free survival rate approached to $~ 95 \%$, and 3-year OS rate approached $90 \%$ after photon-based IMRT (7). Only $22.7 \%$ of their patients achieved R0/1 resection. These findings were echoed by other retrospective series, which showed combination of surgical resection and radiotherapy is superior to single treatment $(2,24,27,29,31)$.

Definitive radiation therapy has been used for unresectable disease and in-operable patients. In a retrospective study reported by Yin et al., most patients presented with inoperable or unresectable disease. The authors reported the 5 -year OS and local control rates of $50 \%$ and $63 \%$, respectively, after radiotherapy alone to a definitive dose of 70 Gy (8). Other studies showed that regardless of the resectability of the primary tumor, the 5-year OS rates range between $20 \%$ and $54 \%$ after photon-based radiotherapy alone $(2,8,9,31,35)$.

PBRT with proton, carbon-ion, or their combination, seems to be highly effective in the management of ONB, whether used definitively or adjuvantly, based on published literatures. Liermann et al. reported a favorable 4-year LRFS of $91 \%$ using CIRT or IMRT+CIRT in radiation naïve patients, although more than half of patients had biopsy or R2 resection (36). In a retrospective study reported from Japan, $57 \%$ of patients did not received surgery for their lesions, the 5 -years OS and LPFS rates reached $93 \%$ and $84 \%$ after PRT (37). The use of PBRT as a single modality treatment was also retrospectively investigated for inoperable ONB. Nakamura et al. reported 
remarkable outcomes of $42 \mathrm{ONB}$ patents treated with PRT without surgery. The 5-year OS and PFS rates was between $76-100 \%$ and $39-80 \%$, respectively, for patients with Kadish A, B and C disease (38). In a more recently published multi-institutional study, researchers from Japan reported LC and OS rates of $83 \%$ and $70.3 \%$, respectively, in a group of 21 patients with T4N0M0 ONB treated with CIRT without surgery. Only 3 (14.3\%) patients experienced recurrence within PTV (39). Our study showed that only 1 patient with Kadish C disease developed local-regional progression after PRT in combination with CIRT boost, with a 2 -year LRPFS of $87.5 \%$.

The effectiveness of chemotherapy in the management of ONB is debatable and no standard regimen has been acknowledged (10), the potential value of chemotherapy needs to be investigated for advanced ONB. In a retrospectively study of 15 patients reported from M.D. Anderson, Su $e t$ al. reported a response rate of $68 \%$ to induction chemotherapy and observed improvements in OS and disease-free survival (DFS) in patients who achieved complete response (40). In contrast, Miller et al. failed to demonstrate the effectiveness of concurrent or adjuvant chemotherapy, used with photon-based radiotherapy, on OS or recurrence-free survival (RFS) (41). However, the 5 -year OS was $<30 \%(9,27)$ after chemotherapy alone for ONB patients. In our study, the response to chemotherapy was not universal and was seen in close to $30 \%$ of the 7 the patients received neoadjuvant chemotherapy. The relatively low response rate was due to, at least in part, heterogenous chemotherapy regimens used prior to the referral of patients to PBRT. Further investigation on the regimen as well as the optimal schedule of chemotherapy used in combination with radiation therapy including PBRT is needed.

The small number of our series and the relatively short follow-up time made comparison with historical data difficult. Nevertheless, only one patient developed local recurrence after high-dose PBRT in our study. Another key pitfall of our study is its retrospective nature, although all consecutive patients were included in this analysis and were treated in a relatively uniform fashion using PBRT in terms of field arrangement and dose/fractionation. As far as we know, no prospective investigation on the use of PBRT for ONB has been initiated, and most published literatures on the use of PBRT for ONB were non-comparative (i.e., only presented the outcomes of a single cohort or arm of patients) and originated from single institutions, comparison of the results after PBRT versus those after photon-based IMRT, preferable in a prospective fashion, is needed. Unfortunately, a randomized trial that compares PBRT versus photon based IMRT using survival outcome as the primary endpoint will probably require hundreds of patients thus will be high impractical due to the rarity of the disease. Our upcoming article will publish the results of a retrospective analysis of $52 \mathrm{ONB}$ patients treated with photon-beam IMRT (7). A comparison between the effectiveness and toxicity profile of PBRT versus IMRT using propensity-score matching methods is being planned.

\section{Conclusions}

Intensity modulated PBRT, used either adjuvantly or definitively, appears efficacious and safe in the management of ONB and produced a relatively favorable OS and localregional control at 2 years. Long-term efficacy and safety profile await longer follow-up. Further investigations, preferably in prospective fashion, will facilitate the understanding of the effectiveness of multimodality treatment for patients with locally advanced ONB.

\section{Acknowledgments}

Funding: This study was supported by grant from the Scientific Research projects of the Shanghai Science and Technology Committee (grant No. 19411951000).

\section{Footnote}

Reporting Checklist: The authors have completed the STROBE reporting checklist. Available at http://dx.doi. org/10.21037/atm-19-4790

Data Sharing Statement: Available at http://dx.doi. org/10.21037/atm-19-4790

Conflicts of Interest: All authors have completed the ICMJE uniform disclosure form (available at http://dx.doi. org/10.21037/atm-19-4790). The authors have no conflicts of interest to declare.

Ethical Statement: The authors are accountable for all aspects of the work in ensuring that questions related to the accuracy or integrity of any part of the work are appropriately investigated and resolved. The study was conducted in accordance with the Declaration of Helsinki (as revised in 2013). This retrospective study was approved by the institutional review board (IRB) of the SPHIC (IRB No. 
191205EXP-01). All patients obtained written informed consent before enrolling in this study. Written informed consent was obtained from the patient for publication of this study and any accompanying images. A copy of the written consent is available for review by the Editor-inChief of this journal.

Open Access Statement: This is an Open Access article distributed in accordance with the Creative Commons Attribution-NonCommercial-NoDerivs 4.0 International License (CC BY-NC-ND 4.0), which permits the noncommercial replication and distribution of the article with the strict proviso that no changes or edits are made and the original work is properly cited (including links to both the formal publication through the relevant DOI and the license). See: https://creativecommons.org/licenses/by-nc-nd/4.0/.

\section{References}

1. Broich G, Pagliari A, Ottaviani F. Esthesioneuroblastoma: a general review of the cases published since the discovery of the tumour in 1924. Anticancer Res 1997;17:2683-706.

2. Dulguerov P, Allal AS, Calcaterra TC.

Esthesioneuroblastoma: a meta-analysis and review. Lancet Oncol 2001;2:683-90.

3. Ferlito A, Rinaldo A, Rhys-Evans PH. Contemporary clinical commentary: esthesioneuroblastoma: an update on management of the neck. Laryngoscope 2003;113:1935-8.

4. Yin Z, Wang Y, Wu Y, et al. Age distribution and agerelated outcomes of olfactory neuroblastoma: a populationbased analysis. Cancer Manag Res 2018;10:1359-64.

5. Diaz EM Jr, Johnigan RH 3rd, Pero C, et al. Olfactory neuroblastoma: the 22-year experience at one comprehensive cancer center. Head Neck 2005;27:138-49.

6. Gruber G, Laedrach K, Baumert B, et al. Esthesioneuroblastoma: irradiation alone and surgery alone are not enough. Int J Radiat Oncol Biol Phys 2002;54:486-91.

7. Bao $\mathrm{C}, \mathrm{Hu} \mathrm{W}, \mathrm{Hu} \mathrm{J}$, et al. Intensity-modulated radiation therapy for esthesioneuroblastoma: 10-year experience of a single institute. Front Oncol 2020;10:1158.

8. Yin ZZ, Gao L, Luo JW, et al. Long-term outcomes of patients with esthesioneuroblastomas: A cohort from a single institution. Oral Oncol 2016;53:48-53.

9. Yuan Y, Ye J, Qiu H, et al. Exploration of the optimal treatment regimes for Esthesioneuroblastoma: a single center experience in China. J Cancer 2018;9:174-81.

10. Sheehan J PREIWH, ed. Youmans and Winn Neurological
Surgery E-Book: Elsevier Health Sciences; 2016:1284-92.

11. Khan FM. The physics of radiation therapy. 4th ed. Philadelphia: Lippincott Williams \& Wilkins; 2009.

12. Amirul Islam M, Yanagi T, Mizoe JE, et al. Comparative study of dose distribution between carbon ion radiotherapy and photon radiotherapy for head and neck tumor. Radiat Med 2008;26:415-21.

13. van de Water TA, Bijl HP, Schilstra C, et al. The potential benefit of radiotherapy with protons in head and neck cancer with respect to normal tissue sparing: a systematic review of literature. Oncologist 2011;16:366-77.

14. Frank SJ, Cox JD, Gillin M, et al. Multifield optimization intensity modulated proton therapy for head and neck tumors: a translation to practice. Int J Radiat Oncol Biol Phys 2014;89:846-53.

15. Frank SJ, Cox JD, Gillin M, et al. Intensity modulated proton therapy for head-and-neck cancer: the first clinical experience. Int J Radiat Oncol Biol Phys 2012;84:S475-6.

16. Elsasser T, Kramer M, Scholz M. Accuracy of the local effect model for the prediction of biologic effects of carbon ion beams in vitro and in vivo. Int $\mathrm{J}$ Radiat Oncol Biol Phys 2008;71:866-72.

17. Jones B. A Simpler Energy Transfer Efficiency Model to Predict Relative Biological Effect for Protons and Heavier Ions. Front Oncol 2015;5:184.

18. Kanai T, Endo M, Minohara S, et al. Biophysical characteristics of HIMAC clinical irradiation system for heavy-ion radiation therapy. Int J Radiat Oncol Biol Phys 1999;44:201-10.

19. Tsujii H, Kamada T, Baba M, et al. Clinical advantages of carbon-ion radiotherapy. New Journal of Physics 2008;10:075009.

20. Emami B, Lyman J, Brown A, et al. Tolerance of normal tissue to therapeutic irradiation. Int J Radiat Oncol Biol Phys 1991;21:109-22.

21. Koto M. Skull base and upper cervical spine tumors. Carbon-Ion Radiotherapy. Springer; 2014. p. 155-61.

22. Kadish S, Goodman M, Wang CC. Olfactory neuroblastoma. A clinical analysis of 17 cases. Cancer 1976;37:1571-6.

23. Morita A, Ebersold MJ, Olsen KD, et al. Esthesioneuroblastoma: prognosis and management. Neurosurgery 1993;32:706-14; discussion 714-5.

24. Carey RM, Godovchik J, Workman AD, et al. Patient, disease, and treatment factors associated with overall survival in esthesioneuroblastoma. Int Forum Allergy Rhinol 2017;7:1186-94.

25. Fu TS, Monteiro E, Muhanna N, et al. Comparison 
of outcomes for open versus endoscopic approaches for olfactory neuroblastoma: A systematic review and individual participant data meta-analysis. Head Neck 2016;38 Suppl 1:E2306-16.

26. Joshi RR, Husain Q, Roman BR, et al. Comparing Kadish, TNM, and the modified Dulguerov staging systems for esthesioneuroblastoma. J Surg Oncol 2019;119:130-42.

27. Orton A, Boothe D, Evans D, et al. Esthesioneuroblastoma: A Patterns-of-Care and Outcomes Analysis of the National Cancer Database. Neurosurgery 2018;83:940-7.

28. Ow TJ, Hanna EY, Roberts DB, et al. Optimization of long-term outcomes for patients with esthesioneuroblastoma. Head Neck 2014;36:524-30.

29. Ozsahin M, Gruber G, Olszyk O, et al. Outcome and prognostic factors in olfactory neuroblastoma: a rare cancer network study. Int J Radiat Oncol Biol Phys 2010;78:992-7.

30. Kim N, Lee CG, Kim EH, et al. Patterns of failures after surgical resection in olfactory neuroblastoma. J Neurooncol 2019;141:459-66.

31. Platek ME, Merzianu M, Mashtare TL, et al. Improved survival following surgery and radiation therapy for olfactory neuroblastoma: analysis of the SEER database. Radiat Oncol 2011;6:41.

32. Harvey RJ, Nalavenkata S, Sacks R, et al. Survival outcomes for stage-matched endoscopic and open resection of olfactory neuroblastoma. Head Neck 2017;39:2425-32.

33. Malouf GG, Casiraghi O, Deutsch E, et al. Lowand high-grade esthesioneuroblastomas display a

Cite this article as: $\mathrm{Hu}$ W, Hu J, Gao J, Yang J, Qiu X, Kong L, $\mathrm{Lu}$ JJ. Intensity-modulated particle beam radiation therapy in the management of olfactory neuroblastoma. Ann Transl Med 2020;8(15):926. doi: 10.21037/atm-19-4790 distinct natural history and outcome. Eur J Cancer 2013;49:1324-34.

34. Saade RE, Hanna EY, Bell D. Prognosis and biology in esthesioneuroblastoma: the emerging role of Hyams grading system. Curr Oncol Rep 2015;17:423.

35. Hollen TR, Morris CG, Kirwan JM, et al.

Esthesioneuroblastoma of the nasal cavity. Am J Clin Oncol 2015;38:311-4.

36. Liermann J, Syed M, Held T, et al. Advanced Radiation Techniques in the Treatment of Esthesioneuroblastoma: A 7-Year Single-Institution's Clinical Experience. Cancers (Basel) 2018;10:457.

37. Nishimura H, Ogino T, Kawashima M, et al. Proton-beam therapy for olfactory neuroblastoma. Int J Radiat Oncol Biol Phys 2007;68:758-62.

38. Nakamura N, Zenda S, Tahara M, et al. Proton beam therapy for olfactory neuroblastoma. Radiother Oncol 2017;122:368-72.

39. Suefuji H, Koto M, Demizu Y, et al. A Retrospective Multicenter Study of Carbon Ion Radiotherapy for Locally Advanced Olfactory Neuroblastomas. Anticancer Res 2018;38:1665-70.

40. Su SY, Bell D, Ferrarotto R, et al. Outcomes for olfactory neuroblastoma treated with induction chemotherapy. Head Neck 2017;39:1671-9.

41. Miller KC, Marinelli JP, Van Gompel JJ, et al. Utility of adjuvant chemotherapy in patients receiving surgery and adjuvant radiotherapy for primary treatment of esthesioneuroblastoma. Head Neck 2019;41:1335-41. 